\title{
Fourth Moment of the Riemann Zeta-function with a Shift along the Real Line
}

\author{
Shun SHIMOMURA
}

Keio University

\begin{abstract}
For the Riemann zeta-function we consider the fourth moment whose square part admits a shift along the real line. Asymptotic formulas are obtained for the shift parameter around 0 and for the one in unbounded regions.
\end{abstract}

\section{Introduction}

The asymptotic formula for the fourth moment of the Riemann zeta-function

$$
\mathcal{I}_{4}(T):=\int_{0}^{T}\left|\zeta\left(\frac{1}{2}+i t\right)\right|^{4} d t=\frac{1}{2 \pi^{2}} T(\log T)^{4}+O\left(T(\log T)^{3}\right)
$$

was proved by Ingham [4]. Ramachandra [12], [13] gave a simplified proof of this formula, in which the identity of Montgomery and Vaughan [9] (see also [6, §5.3]) plays an essential role. A precise representation with lower order terms of the form

$$
\mathcal{I}_{4}(T)=\sum_{j=0}^{4} c_{j} T(\log T)^{j}+O\left(T^{7 / 8+\varepsilon}\right)
$$

was presented by Heath-Brown [2]. For a weighted fourth moment Motohashi established an explicit formula based on spectral theory of automorphic forms, which brought about the improvement $\ll T^{2 / 3}(\log T)^{8}$ on the error term above (cf. [10]).

The second moment with shift parameters

$$
\int_{1}^{T} \zeta\left(\frac{1}{2}+a+i t\right) \zeta\left(\frac{1}{2}-b-i t\right) d t
$$

was also treated in [4], and Bettin [1] gave an asymptotic formula for unbounded shifts near the critical line. A shifted fourth moment was discussed in [10], and an asymptotic formula

Received April 25, 2012; revised October 16, 2012

Mathematics Subject Classification: 11M06

Key words and phrases: Riemann zeta-function, fourth moment 
for a twisted fourth moment presented by Hughes and Young [3] contains small shift parameters $\ll(\log T)^{-1}$. Concerning shifted moments of another type Kösters [8] pointed out an interesting occurrence of the sine kernel for random matrix ensembles. For a shifted fourth moment of the form

$$
\int_{0}^{T}\left|\zeta\left(\frac{1}{2}+i t\right) \zeta\left(\frac{1}{2}+a+i t\right)\right|^{2} d t
$$

with $a i=\lambda \in \mathbf{R}$ we [15] studied the asymptotic behaviour in the shift range $|\lambda| \ll$ $\exp \left((\log T)^{3 / 8-\varepsilon}\right)$, in which the sine kernel appears if $|\lambda| \ll(\log T)^{-\beta}$ with $0<\beta \leq 1 / 2$.

In this paper we consider this type of fourth moment for $a \in \mathbf{R}$. Asymptotic formulas are obtained for $a$ around 0 and for $a$ in ranges unbounded as $T \rightarrow \infty$. In deriving them we apply the method of Ramachandra to $\zeta(s) \zeta(s+a)$. In addition to the identity of Montgomery and Vaughan we need a summation formula related to $\sigma_{-a}(n)=\sum_{d \mid n} d^{-a}$, which is given in Section 3. Our results are proved in Section 6 by using the facts described in Sections 4 and 5 . Throughout this paper $\varepsilon$ denotes an arbitrary small positive number permitted to vary in each appearance.

\section{Main results}

For $a \in \mathbf{R}$ and $T \gg 1$, let

$$
\Psi(a, T):=\frac{1}{T} \int_{1}^{T}\left|\zeta\left(\frac{1}{2}+i t\right) \zeta\left(\frac{1}{2}+a+i t\right)\right|^{2} d t
$$

Our results are stated as follows:

THEOREM 2.1. As $|a|+(\log T)^{-1} \rightarrow 0$,

$$
\Psi(a, T)=3 \pi^{-2} a^{-4} \phi(a \log T)\left(1+O\left(|a|+(\log T)^{-1}\right)\right)
$$

with $\phi(y):=-2+y+4 e^{-y}-(2+y) e^{-2 y}$, the implied constant being absolute.

THEOREM 2.2. Suppose that $\theta(a):=|2 \pi a|^{2|a|}+|a|^{-1}=o(\log T)$. If $a<0$,

$$
\Psi(a, T)=\frac{(2 \pi)^{2 a} \zeta(1-2 a) \zeta(1-a)^{2}}{(1-2 a) \zeta(2-2 a)} T^{-2 a} \log T\left(1+O\left(\theta(a)(\log T)^{-1}\right)\right)
$$

and if $a>0$,

$$
\Psi(a, T)=\frac{\zeta(1+2 a) \zeta(1+a)^{2}}{\zeta(2+2 a)} \log T\left(1+O\left(\theta(a)(\log T)^{-1}\right)\right) .
$$

Here the implied constants are absolute. 
REMARK 2.1. Since $y^{-4} \phi(y)=1 / 6+O(y)$ around $y=0$, Theorem 2.1 gives $\Psi(0, T)=\left(2 \pi^{2}\right)^{-1}(\log T)^{4}\left(1+O\left((\log T)^{-1}\right)\right)$ as $(\log T)^{-1} \rightarrow 0$, which coincides with the result of Ingham [4].

REMARK 2.2. Let $0<\beta \leq 1$. For $|a| \ll(\log T)^{-\beta}$ (respectively, $(\log T)^{-1+\beta} \ll$ $|a| \ll 1)$ the error term in Theorem 2.1 (respectively, Theorem 2.2) is $\ll(\log T)^{-\beta}$. The formulas in Theorem 2.2 are significant for $a$ in regions unbounded as $T \rightarrow \infty$. Indeed, if $(\log T)^{-\varepsilon} \ll|a| \ll(\log \log T)^{1-\varepsilon}$, then each error term is $\ll(\log T)^{-1+\varepsilon}$.

\section{Sums related to $\sigma_{-a}(n)$}

Recall the formula

$$
Z(a, s):=\frac{\zeta(s) \zeta(s+a)^{2} \zeta(s+2 a)}{\zeta(2 s+2 a)}=\sum_{n=1}^{\infty} \frac{\sigma_{-a}(n)^{2}}{n^{s}}
$$

for $\operatorname{Re}(s)>\max \{1,1-2 a\}$ (cf. [6, p. 37] or [16, (1.3.3)]). The function $\left(x^{s} / s\right) Z(a, s)$ with $x>0$ possesses poles at least at $s=1,1-a, 1-2 a$ if $|a|<1 / 2$, at $s=1$ if $a \geq 1 / 2$, and at $s=1-2 a$ if $a \leq-1 / 2$. If $a \neq 0$, the residues are, respectively,

$$
\begin{aligned}
R_{1}:= & \frac{\zeta(1+2 a) \zeta(1+a)^{2}}{\zeta(2+2 a)} x \\
R_{1-a}:= & \frac{\zeta(1-a) \zeta(1+a)}{\zeta(2)(1-a)} x^{1-a} \\
& \times\left(\log x-\frac{1}{1-a}+\frac{\zeta^{\prime}}{\zeta}(1-a)+\frac{\zeta^{\prime}}{\zeta}(1+a)-2 \frac{\zeta^{\prime}}{\zeta}(2)+2 \gamma_{\mathrm{e}}\right), \\
R_{1-2 a}:= & \frac{\zeta(1-2 a) \zeta(1-a)^{2}}{(1-2 a) \zeta(2-2 a)} x^{1-2 a},
\end{aligned}
$$

where $\gamma_{\mathrm{e}}$ is the Euler constant. These three quantities are also meromorphic in $a \in \mathbf{C}$, and are holomorphic around $a= \pm 1 / 2$. Furthermore the sum of them is holomorphic around $a=0$. Indeed, for $0<|a|<1 / 16$

$$
R_{1}+R_{1-a}+R_{1-2 a}=\frac{1}{2 \pi i} \int_{\Delta} \frac{x^{s}}{s} Z(a, s) d s
$$

with the positively oriented circle $\Delta:|s-1|=1 / 4$, where the integrand $\left(x^{s} / s\right) Z(a, s)$ restricted to $\Delta$ is holomorphic around $a=0$. The holomorphy of the sum around $a=0$ may also be checked by a direct computation of the Laurent series expansion (cf. Section 4.1). For $x \geq 2$, restricting to $a \in \mathbf{R}$ again, we set

$$
\Lambda(a, x):= \begin{cases}x^{-1} R_{1-2 a} & \text { if } a \leq-1 / 2, \\ x^{-1}\left(R_{1}+R_{1-a}+R_{1-2 a}\right) & \text { if }-1 / 2<a<1 / 2, \\ x^{-1} R_{1} & \text { if } a \geq 1 / 2,\end{cases}
$$


which is continuous in $a \in \mathbf{R} \backslash\{ \pm 1 / 2\}$.

PROPOSITION 3.1. For $a \in \mathbf{R}, \sum_{n \leq x} \sigma_{-a}(n)^{2}=x \Lambda(a, x)+E_{a}(x)$, the error term $E_{a}(x)$ satisfying $\ll x^{1 / 2-2 a+\varepsilon}$ if $a \leq 0$, and $\ll x^{1 / 2+\varepsilon}$ if $a \geq 0$. Here the implied constant depends on $\varepsilon$ only.

For each $a$, better error estimates are known [5], [11], [14]. In particular, $E_{a}(x) \ll$ $x^{1 / 2-2 a / 3}(\log x)^{5}$ for $0<a \leq 3 / 8$ (cf. [11]), while $E_{0}(x) \ll x^{1 / 2}(\log x)^{5} \log \log x(\mathrm{cf}$. [14]). In the proof below we put emphasis on the uniformity in $a$.

PROOF. Let $\delta$ be a given small positive number. First we consider the case $0 \leq a \leq$ $1 / 4-\delta$. Let $\omega$ be a number such that $\omega \geq x^{1 / 2}$. By Perron's formula,

$$
\sum_{n \leq x} \sigma_{-a}(n)^{2}=\frac{1}{2 \pi i} \int_{1+\delta-i \omega}^{1+\delta+i \omega} \frac{x^{s}}{s} Z(a, s) d s+O\left(x^{1 / 2+\delta}\right)
$$

with $s=\sigma+i t$. The poles of the integrand inside the rectangle with vertices $1+\delta \pm i \omega$, $1 / 2 \pm i \omega$ are $s=1,1-a, 1-2 a$, and hence

$$
I_{1+\delta-i \omega}^{1+\delta+i \omega}-I_{1 / 2+i \omega}^{1+\delta+i \omega}-I_{1 / 2-i \omega}^{1 / 2+i \omega}+I_{1 / 2-i \omega}^{1+\delta-i \omega}=R_{1}+R_{1-a}+R_{1-2 a}=x \Lambda(a, x),
$$

provided that $a \neq 0$, where

$$
I_{z_{-}}^{z_{+}}:=\frac{1}{2 \pi i} \int_{z_{-}}^{z_{+}} \frac{x^{s}}{s} Z(a, s) d s .
$$

This equality is also valid for $a=0$, because both sides are holomorphic around $a=0$. Hence

$$
\sum_{n \leq x} \sigma_{-a}(n)^{2}=x \Lambda(a, x)+I_{0}-I_{-}+I_{+}+O\left(x^{1 / 2+\delta}\right)
$$

with $I_{+}=-\overline{I_{-}}:=I_{1 / 2+i \omega}^{1+\delta+i \omega}$ and $I_{0}:=I_{1 / 2-i \omega}^{1 / 2+i \omega}$. To evaluate $I_{+}$set

$$
g(t):=\int_{1 / 2}^{1+\delta}\left|\zeta(\sigma+i t) \zeta(\sigma+a+i t)^{2} \zeta(\sigma+2 a+i t)\right| d \sigma .
$$

Since

$$
\begin{aligned}
\int_{x^{1 / 2}}^{2 x^{1 / 2}} g(t) d t \ll & \int_{1 / 2}^{1+\delta}\left(\int_{x^{1 / 2}}^{2 x^{1 / 2}}|\zeta(\sigma+i t)|^{4} d t\left(\int_{x^{1 / 2}}^{2 x^{1 / 2}}|\zeta(\sigma+a+i t)|^{4} d t\right)^{2}\right. \\
& \left.\times \int_{x^{1 / 2}}^{2 x^{1 / 2}}|\zeta(\sigma+2 a+i t)|^{4} d t\right)^{1 / 4} d \sigma \ll x^{1 / 2+\delta},
\end{aligned}
$$

we may choose $\omega \in\left[x^{1 / 2}, 2 x^{1 / 2}\right]$ so that $g(\omega) \ll x^{\delta}$. Then, observing $\zeta(2 s)^{-1} \ll \log t$ for 
$1 / 2 \leq \sigma$, we have $I_{+} \ll x^{1+\delta} \omega^{-1} \cdot \log x \cdot g(\omega) \ll x^{1 / 2+3 \delta}$. Furthermore

$$
\begin{aligned}
I_{0} & \ll x^{1 / 2} \log \omega\left(\int_{1}^{\omega}\left|\zeta(1 / 2+i t) \zeta(1 / 2+a+i t)^{2} \zeta(1 / 2+2 a+i t)\right| \frac{d t}{t}+\delta^{-1}\right) \\
& \ll x^{1 / 2} \log \omega\left(J(0)^{1 / 4} J(a)^{1 / 2} J(2 a)^{1 / 4}+\delta^{-1}\right),
\end{aligned}
$$

where

$$
J(v):=\int_{1}^{\omega}|\zeta(1 / 2+v+i t)|^{4} \frac{d t}{t} .
$$

Integrating by parts, we have, uniformly in $v \geq 0$,

$$
J(v) \ll \frac{1}{\omega} \int_{1}^{\omega}|\zeta(1 / 2+v+i t)|^{4} d t+\int_{1}^{\omega} \int_{1}^{t}|\zeta(1 / 2+v+i \rho)|^{4} d \rho \frac{d t}{t^{2}} \ll \omega^{\delta} .
$$

This gives $I_{0} \ll x^{1 / 2+2 \delta}$, the implied constant depending on $\delta$ only. Thus we obtain $\sum_{n \leq x} \sigma_{-a}(n)^{2}=x \Lambda(a, x)+O\left(x^{1 / 2+3 \delta}\right)$ for $0 \leq a \leq 1 / 4-\delta$. Next suppose that $1 / 4-\delta \leq a \leq 1 / 2-4 \delta$. The poles are arrayed as $1-2 a<1 / 2+3 \delta<1-a<1$, and hence $x \Lambda(a, x)=R_{1}+R_{1-a}+O\left(x^{1 / 2+3 \delta}\right)$. In (3.2), shift of the path of integration to $\sigma=1 / 2+3 \delta$ takes the right-hand side to

$$
x \Lambda(a, x)+I_{1 / 2+3 \delta-i \omega}^{1 / 2+3 \delta+i \omega}+I_{1 / 2+3 \delta+i \omega}^{1+\delta+i \omega}+\overline{I_{1 / 2+3 \delta+i \omega}^{1+\delta+i \omega}}+O\left(x^{1 / 2+3 \delta}\right) .
$$

Then $I_{1 / 2+3 \delta+i \omega}^{1+\delta+i \omega} \ll x^{1 / 2+3 \delta}$ and $I_{1 / 2+3 \delta-i \omega}^{1 / 2+3 \delta+i \omega} \ll x^{1 / 2+5 \delta}$, which implies the formula with the error term $\ll x^{1 / 2+5 \delta}$. If $a \geq 1 / 2-4 \delta$, then $x \Lambda(a, x)=R_{1}+O\left(x^{1 / 2+5 \delta}\right)$, since $1-a<1 / 2+5 \delta$. Shifting the path of integration to $\sigma=1 / 2+5 \delta$, and noting the bound of $|\zeta(1 / 2+a+i t)|$ uniform in $a \geq 1$, we similarly obtain the error term $\ll x^{1 / 2+7 \delta}$ with the implied constant independent of $a$. Therefore $E_{a}(x) \ll x^{1 / 2+\varepsilon}$ for $a \geq 0$. The formula for $a \leq 0$ is an immediate consequence of the fact

$$
\sum_{n \leq x} \sigma_{-a}(n)^{2}=\sum_{n \leq x} n^{-2 a} \sigma_{a}(n)^{2}=x^{-2 a} \sum_{n \leq x} \sigma_{a}(n)^{2}+2 a \int_{1}^{x} \xi^{-2 a-1}\left(\sum_{n \leq \xi} \sigma_{a}(n)^{2}\right) d \xi .
$$

Thus we obtain the proposition.

We need the following estimate as well (for the summation formula see [7]).

Proposition 3.2. For $a \in \mathbf{R}, \sum_{n \leq x} \sigma_{-a}(n) \ll(1+|a|)\left|x\left(1-x^{-a}\right) / a\right|$, the implied constant being absolute.

ProOF. Note that $\sum_{n \leq x} \sigma_{-a}(n)=\sum_{n \leq x} \sum_{d \mid n} d^{-a}=\sum_{m \leq x} \sum_{d \leq x / m} d^{-a}$, where the last sum satisfies, for $|a| \leq 1 / 2$,

$$
\ll x^{1-a} \sum_{m \leq x} m^{a-1} \ll x^{1-a}\left(1+\int_{1}^{x} \xi^{a-1} d \xi\right)=x^{1-a}+\frac{x}{a}\left(1-x^{-a}\right) .
$$


Using $\left|a x^{-a} /\left(1-x^{-a}\right)\right|=\left|X e^{-X} /\left(1-e^{-X}\right)\right|(\log x)^{-1} \ll(1+|X|)(\log x)^{-1} \ll 1+|a|$ with $X=a \log x$, we obtain the desired estimate for $|a| \leq 1 / 2$. Since, for $a \geq 1 / 2$, $\sum_{n \leq x} \sigma_{-a}(n) \leq \sum_{n \leq x} \sigma_{-1 / 2}(n) \ll x$, the lemma is valid for $a \geq-1 / 2$. Hence, for $a \leq-1 / 2$ as well, we have $\sum_{n \leq x} \sigma_{-a}(n)=\sum_{n \leq x} n^{-a} \sigma_{a}(n) \leq x^{-a} \sum_{n \leq x} \sigma_{a}(n) \ll$ $(1+|a|)\left|x\left(1-x^{-a}\right) / a\right|$. This completes the proof.

\section{Lemmas}

Set, for $x \geq 1$ and for $a \in \mathbf{R}$,

$$
\Phi(a, x):=\int_{1}^{x} \xi^{-1} \Lambda(a, \xi) d \xi
$$

Let $N$ be a positive integer such that $x^{1 / 4} \leq 2^{-N} x \leq 2 x^{1 / 4}$. Define related sums by

$$
\begin{gathered}
\Phi_{N}(a, x):=\sum_{n=0}^{N} 2^{-n} \Phi\left(a, 2^{-n} x\right), \quad|\Lambda|_{N}(a, x):=\sum_{n=0}^{N} 2^{-n}\left|\Lambda\left(a, 2^{-n} x\right)\right|, \\
\widetilde{\Phi}_{N}(a, x):=\sum_{n=0}^{N} 2^{(2 a-1) n} x^{-2 a} \Phi\left(-a, 2^{-n} x\right) .
\end{gathered}
$$

Then we have the following, in which the implied constants are absolute.

LEMMA 4.1. As $|a|+(\log x)^{-1} \rightarrow 0$,

$$
\begin{aligned}
& \Phi_{N}(a, x)=6 \pi^{-2} a^{-4} \psi(a, x)\left(1+O\left(|a|+(\log x)^{-1}\right)\right) \gg \log x, \\
& \widetilde{\Phi}_{N}(a, x)=6 \pi^{-2} a^{-4} x^{-2 a} \psi(-a, x)\left(1+O\left(|a|+(\log x)^{-1}\right)\right),
\end{aligned}
$$

where $\psi(a, x):=-5 / 2+a \log x+2(1+a \log x) x^{-a}+x^{-2 a} / 2$.

In the lemma above $\hat{\psi}(a, x):=a^{-4} \psi(a, x)$ satisfies $\hat{\psi}(0, x)=(\log x)^{4} / 12$ (cf. (4.5) in the proof of Lemma 4.1).

LeMmA 4.2. Let $a<0$. As $|a \log x|^{-1} \rightarrow 0$,

$$
\widetilde{\Phi}_{N}(a, x)=\frac{\Lambda_{0}(-a)}{1-2^{2 a-1}} x^{-2 a} \log x\left(1+O\left(|a \log x|^{-1}\right)\right),
$$

where $\Lambda_{0}(a):=\zeta(1+2 a) \zeta(1+a)^{2} \zeta(2+2 a)^{-1}$.

LEMMA 4.3. As $|a|+(\log x)^{-1} \rightarrow 0,|\Lambda|_{N}(a, x) \ll \Phi_{N}(a, x)\left(|a|+(\log x)^{-1}\right)$. Furthermore, if $a<0$, then $|\Lambda|_{N}(a, x) \ll \widetilde{\Phi}_{N}(a, x)(\log x)^{-1}$ as $|a \log x|^{-1} \rightarrow 0$.

Let $\tau$ be a sufficiently large positive number. Set

$$
\Lambda^{*}(a, x):=\left(a^{2}+1\right) \Lambda(a, x) .
$$


LEMMA 4.4. (1) For $a \in \mathbf{R}$,

$$
\sum_{n \leq \tau} \frac{\sigma_{-a}(n)^{2}}{n}=\Phi(a, \tau)+O\left(\Lambda^{*}(a, \tau)\right)
$$

the implied constant being absolute.

(2) For $a \geq 0$, if $\mu-1 \geq c_{0}>0$ (respectively, $\mu-1 \leq-c_{0}<0$ ),

$$
\sum_{n>\tau} \frac{\sigma_{-a}(n)^{2}}{n^{\mu}}\left(\text { respectively, } \sum_{n \leq \tau} \frac{\sigma_{-a}(n)^{2}}{n^{\mu}}\right) \ll \tau^{1-\mu} \Lambda^{*}(a, \tau),
$$

the implied constant depending on $c_{0}$ only.

(3) For $a<0$, if $\mu+2 a-1 \geq c_{0}>0$ (respectively, $\mu-1 \leq-c_{0}<0$ ), we have the same estimate as in (2) with the implied constant depending on $c_{0}$ only.

LEMMA 4.5. For $a \in \mathbf{R}$ and for $\sigma-1-\max \{0, a\} \geq c_{0}>0$ (respectively, $0<\sigma \leq$ $\left.1-c_{0}<1\right)$,

$$
\sum_{n>\tau} \frac{\sigma_{a}(n)}{n^{\sigma}}\left(\text { respectively, } \sum_{n \leq \tau} \frac{\sigma_{a}(n)}{n^{\sigma}}\right) \ll\left(1+a^{2}\right) \tau^{1-\sigma}\left|\frac{1-\tau^{a}}{a}\right|,
$$

the implied constant depending on $c_{0}$ only.

These lemmas are verified in the remaining part of this section.

4.1. $\Lambda(a, x)$ and $\Phi(a, x)$ around $a=0$. The function $\Lambda(a, x)$ is holomorphic for $|a|<1 / 2$. The Laurent series expansion of $\zeta(1+a)$ gives the expression

$$
\begin{aligned}
\Lambda(a, x)= & \pi^{-2} l_{3}(a, x)+c_{2}^{1} l_{2}^{1}(a, x)+c_{2}^{2} l_{2}^{2}(a, x)+c_{1}^{1} l_{1}^{1}(a, x)+c_{1}^{2} l_{1}^{2}(a, x) \\
& +g_{0}(a) x^{-a} \log x+g_{1}(a) x^{-a}+g_{2}(a) x^{-2 a}+g_{3}(a) .
\end{aligned}
$$

Here $c_{i}^{j}$ are certain real numbers; $g_{k}(a)$ are holomorphic for $|a|<1 / 2$; and

$$
\begin{gathered}
l_{3}(a, x):=3 a^{-3}\left(1-2 a x^{-a} \log x-x^{-2 a}\right), \\
l_{2}^{1}(a, x):=a^{-2} x^{-a}\left(1-a \log x-x^{-a}\right), \quad l_{2}^{2}(a, x):=a^{-2}\left(1-x^{-a}\right)^{2}, \\
l_{1}^{1}(a, x):=a^{-1} x^{-a}\left(1-x^{-a}\right), \quad l_{1}^{2}(a, x):=a^{-1}\left(1-x^{-2 a}\right) .
\end{gathered}
$$

These functions are holomorphic around $a=0$. Furthermore, for $x \geq 1$, by definition

$$
\begin{aligned}
\Phi(a, x)= & 3 \pi^{-2} a^{-4} \psi(a, x)+c_{2}^{1} l_{2}^{1 *}(a, x)+c_{2}^{2} l_{2}^{2 *}(a, x)+c_{1}^{1} l_{1}^{1 *}(a, x)+c_{1}^{2} l_{1}^{2 *}(a, x) \\
& +g_{0}(a) \eta_{0}(a, x)+g_{1}(a) \eta_{1}(a, x)+g_{2}(a) \eta_{1}(2 a, x)+g_{3}(a) \log x .
\end{aligned}
$$

Here

$$
\begin{gathered}
\psi(a, x)=-5 / 2+a \log x+2(1+a \log x) x^{-a}+x^{-2 a} / 2, \\
l_{2}^{1 *}(a, x):=a^{-3}\left(-1 / 2+a x^{-a} \log x+x^{-2 a} / 2\right),
\end{gathered}
$$




$$
\begin{gathered}
l_{2}^{2 *}(a, x):=a^{-3}\left(-3 / 2+a \log x+2 x^{-a}-x^{-2 a} / 2\right), \\
l_{1}^{1 *}(a, x):=a^{-2}\left(1-x^{-a}\right)^{2} / 2, \quad l_{1}^{2 *}(a, x):=a^{-2}\left(-1 / 2+a \log x+x^{-2 a} / 2\right), \\
\eta_{0}(a, x):=a^{-2}\left(1-a x^{-a} \log x-x^{-a}\right), \quad \eta_{1}(a, x):=a^{-1}\left(1-x^{-a}\right) .
\end{gathered}
$$

4.2. Proof of Lemma 4.1. Note that, for $a<1 / 4$,

$$
\begin{aligned}
& \sum_{n \geq 0}\left(2^{-n}\right)^{1-a} \log \left(2^{-n} x\right)=\frac{\log x}{1-2^{a-1}}-\frac{2^{a-1} \log 2}{\left(1-2^{a-1}\right)^{2}}, \\
& \sum_{n \geq 0} 2^{-n}\left(\left(2^{-n} x\right)^{-a}-1\right)=\frac{x^{-a}-2+2^{a}}{1-2^{a-1}} .
\end{aligned}
$$

For $|a|<1 / 4, x \geq 2$, we have $\sum_{n \geq 0} 2^{-n} \psi\left(a, 2^{-n} x\right)=h(a, a \log x)$, where

$$
\begin{aligned}
h(a, y):= & 2\left(y-a^{\prime}\right)+2 e^{-y}\left(\frac{y}{1-e^{a^{\prime}} / 2}-\frac{a^{\prime} e^{a^{\prime}} / 2}{\left(1-e^{a^{\prime}} / 2\right)^{2}}\right) \\
& +\frac{2\left(e^{-y}-2+e^{a^{\prime}}\right)}{1-e^{a^{\prime}} / 2}+\frac{e^{-2 y}-2+e^{2 a^{\prime}}}{2\left(1-e^{2 a^{\prime}} / 2\right)}
\end{aligned}
$$

with $a^{\prime}:=a \log 2$. Furthermore $\psi(a, x)=h_{0}(a \log x)$ with

$$
h_{0}(y):=-5 / 2+y+2(1+y) e^{-y}+e^{-2 y} / 2 .
$$

Computation of the series expansion leads us to

$$
h(a, y)-2 h_{0}(y) \ll|a|(|y|+|a|)^{3},
$$

provided that $|y| \ll 1,|a|<1 / 4$. The function $h_{0}(y)$ satisfies

$$
h_{0}(y)=\left(y^{4} / 12\right)(1+O(y))
$$

for $|y| \ll 1$; and

$$
h_{0}(y) \gg|y|+e^{-2 y}
$$

for $|y| \gg 1$. If $|y| \ll 1$ and if $|a|<1 / 4$, then, by (4.4) and (4.5), $h(a, y) / h_{0}(y)-2 \ll$ $|a / y|+|a / y|^{4}$. For $y \in \mathbf{R}, h(a, y)-2 h_{0}(y) \ll|a|\left(1+e^{-2 y}\right)$, since $h(0, y) \equiv 2 h_{0}(y)$. Hence, if $|y| \gg 1$ and if $|a|<1 / 4$, then, by (4.6), $h(a, y) / h_{0}(y)-2 \ll|a|$. Thus we have $h(a, y)=2 h_{0}(y)\left(1+O\left(|a|+|a / y|+|a / y|^{4}\right)\right)$ for $|a|<1 / 4$, which implies

$$
\sum_{n \geq 0} 2^{-n} \psi\left(a, 2^{-n} x\right)=h(a, a \log x)=2 h_{0}(a \log x)\left(1+O\left(|a|+(\log x)^{-1}\right)\right)
$$


as $|a|+(\log x)^{-1} \rightarrow 0$. Note that $h_{0}(y)>h_{0}\left(y^{\prime}\right) \geq 0$ if $y>y^{\prime} \geq 0$ or if $y<y^{\prime} \leq 0$. Since $2^{-N} x \asymp x^{1 / 4}$, it follows that

$$
\begin{aligned}
& \sum_{n=0}^{N} 2^{-n} \psi\left(a, 2^{-n} x\right)=h(a, a \log x)-2^{-N-1} h\left(a, a \log \left(2^{-N-1} x\right)\right) \\
& \quad=2 h_{0}(a \log x)\left(1+O\left(|a|+(\log x)^{-1}\right)\right) \gg \min \left\{(a \log x)^{4},|a| \log x\right\}
\end{aligned}
$$

with $h_{0}(a \log x)=\psi(a, x)$. To show the formula for $\Phi_{N}(a, x)$ it remains to evaluate the sums $\sum_{n=0}^{N} 2^{-n} l_{i}^{j *}\left(a, 2^{-n} x\right)$ and $\sum_{n=0}^{N} 2^{-n} \eta_{i}\left(a, 2^{-n} x\right)$. Write $l_{2}^{1 *}(a, x)=a^{-3} \varphi_{2}^{1 *}(a \log x)$, where $\varphi_{2}^{1 *}(y):=-1 / 2+y e^{-y}+e^{-2 y} / 2$ has the properties:

(a) $\left|\varphi_{2}^{1 *}(y)\right|>\left|\varphi_{2}^{1 *}\left(y^{\prime}\right)\right|$ if $y>y^{\prime} \geq 0$ or if $y<y^{\prime} \leq 0$;

(b) $\left|\varphi_{2}^{1 *}(y)\right| \asymp|y|^{3}$ for $|y| \ll 1$;

(c) $\left|\varphi_{2}^{1 *}(y)\right| \ll|y|+e^{-2 y}$ for $|y| \gg 1$.

Then, by (4.5) and (4.6), $\left|\varphi_{2}^{1 *}(y)\right| \ll h_{0}(y)\left(1+|y|^{-1}\right)$ uniformly in $y \in \mathbf{R}$. Since $2^{-n} x>1$ for $n \leq N$, we have

$$
\begin{aligned}
& \sum_{n=0}^{N} 2^{-n} l_{2}^{1 *}\left(a, 2^{-n} x\right) \ll \sum_{n=0}^{N} 2^{-n}|a|^{-3}\left|\varphi_{2}^{1 *}\left(a \log \left(2^{-n} x\right)\right)\right| \\
& \quad \ll|a|^{-3}\left|\varphi_{2}^{1 *}(a \log x)\right| \ll a^{-4} h_{0}(a \log x)\left(|a|+(\log x)^{-1}\right) .
\end{aligned}
$$

The other sums for $l_{i}^{j *}(a, x), \eta_{i}(a, x)$ and $\log x$ admit the same estimates. From (4.7) combined with these estimates the first expression of Lemma 4.1 immediately follows.

To derive the formula for $\widetilde{\Phi}_{N}(a, x)$ we calculate, for $x \geq 2,|a|<1 / 4$,

$$
\begin{gathered}
\Sigma:=\sum_{n \geq 0} 2^{(2 a-1) n} x^{-2 a} \psi\left(-a, 2^{-n} x\right)=\sum_{n \geq 0} 2^{-n} \tilde{\psi}\left(a, 2^{-n} x\right), \\
\tilde{\psi}(a, x):=x^{-2 a} \psi(-a, x)=1 / 2+2(1-a \log x) x^{-a}-(5 / 2+a \log x) x^{-2 a} .
\end{gathered}
$$

Note that $\tilde{\psi}(a, x)=\tilde{h}_{0}(a \log x)$ with $\tilde{h}_{0}(y):=e^{-2 y} h_{0}(-y)$. By (4.2) and (4.3) we have $\Sigma=\tilde{h}(a, a \log x)$ with

$$
\begin{gathered}
\tilde{h}(a, y):=-e^{-2 y}\left(\frac{y}{1-e^{2 a^{\prime}} / 2}-\frac{a^{\prime} e^{2 a^{\prime}} / 2}{\left(1-e^{2 a^{\prime}} / 2\right)^{2}}\right)-\frac{(5 / 2) e^{-2 y}}{1-e^{2 a^{\prime}} / 2} \\
-2 e^{-y}\left(\frac{y}{1-e^{a^{\prime}} / 2}-\frac{a^{\prime} e^{a^{\prime}} / 2}{\left(1-e^{a^{\prime}} / 2\right)^{2}}\right)+\frac{2 e^{-y}}{1-e^{a^{\prime}} / 2}+1
\end{gathered}
$$

satisfying $\tilde{h}(a, y)-2 \tilde{h}_{0}(y) \ll|a|(|a|+|y|)^{3}$ for $|y| \ll 1,|a|<1 / 4$. From this we get $\sum_{n=0}^{N} 2^{-n} \tilde{\psi}\left(a, 2^{-n} x\right)=2 \tilde{h}_{0}(a \log x)\left(1+O\left(|a|+(\log x)^{-1}\right)\right)$. Write $\tilde{l}_{2}^{1 *}(a, x):=$ $x^{-2 a} l_{2}^{1 *}(-a, x)=a^{-3} \tilde{\varphi}_{2}^{1 *}(a \log x)$ with $\tilde{\varphi}_{2}^{1 *}(y):=-1 / 2+y e^{-y}+e^{-2 y} / 2$ admitting the same property as of $\varphi_{2}^{1 *}(y)$. Then it follows that $\sum_{n=0}^{N} 2^{-n} \tilde{l}_{2}^{1 *}\left(a, 2^{-n} x\right) \ll$ 
$a^{-4} \tilde{h}_{0}(a \log x)\left(|a|+(\log x)^{-1}\right)$. The same estimates are verified for the sums related to the remaining $l_{i}^{j *}(-a, x)$. Furthermore, $\tilde{\eta}_{0}(a, x):=x^{-2 a} \eta_{0}(-a, x)=a^{-2} \rho_{0}(a \log x)$ and $\tilde{\eta}_{1}(a, x):=x^{-2 a} \eta_{1}(-a, x)=a^{-1} \rho_{1}(a \log x)$, where $\rho_{0}(y):=e^{-2 y}+y e^{-y}-e^{-y}$ and $\rho_{1}(y):=e^{-y}-e^{-2 y}$, respectively. The functions $\rho_{i}(y)$ satisfy

(a) $\left|\rho_{0}(y)\right| \asymp y^{2}$ and $\left|\rho_{1}(y)\right| \asymp|y|$ for $|y| \ll 1$;

(b) $\left|\rho_{i}(y)\right| \ll e^{-2 y}+|y| e^{-y}$ for $|y| \gg 1$;

(c) $\left|\rho_{i}(y)\right|>\left|\rho_{i}\left(y^{\prime}\right)\right|$ if $y<y^{\prime} \leq 0$;

(d) there exists a positive number $c_{0}^{(i)}$ such that $\left|\rho_{i}(y)\right|>\left|\rho_{i}\left(y^{\prime}\right)\right|$ if $0 \leq y^{\prime}<y \leq c_{0}^{(i)}$ and that $0<\rho_{i}(y) \ll 1$ if $y \geq c_{0}^{(i)}$.

Hence, if $a \log x \leq 0$ or if $a \log x \ll 1$,

$$
\sum_{n=0}^{N} 2^{-n} \tilde{\eta}_{0}\left(a, 2^{-n} x\right) \ll a^{-2}\left|\rho_{0}(a \log x)\right| \ll a^{-4} \tilde{h}_{0}(a \log x)\left(a^{2}+(\log x)^{-2}\right),
$$

and otherwise, $\ll a^{-2} \ll a^{-4} \tilde{h}_{0}(a \log x) \cdot a^{2}$. Combining the facts above, we arrive at the second formula of Lemma 4.1 .

4.3. Proof of Lemma 4.2. By (4.2), for $a<0$,

$$
\sum_{n=0}^{N} 2^{(2 a-1) n} \log \left(2^{-n} x\right)=\frac{\log x}{1-2^{2 a-1}}\left(1+O\left(|a \log x|^{-1}\right)\right)
$$

since $2^{-N} x \asymp x^{1 / 4}$. Recalling (3.1) for $|a|<1 / 2$, we write

$$
\Lambda(a, x)=\Lambda_{0}(a)+\Lambda_{1}(a) x^{-a}+\Lambda_{2}(a) x^{-a} \log x+\frac{\Lambda_{0}(-a)}{1-2 a} x^{-2 a}
$$

with $\Lambda_{2}(a):=\zeta(1-a) \zeta(1+a)(1-a)^{-1} / \zeta(2)$,

$$
\Lambda_{1}(a):=-\frac{\zeta(1-a) \zeta(1+a)}{\zeta(2)(1-a)^{2}}\left(1-(1-a)\left(\frac{\zeta^{\prime}}{\zeta}(1-a)+\frac{\zeta^{\prime}}{\zeta}(1+a)-2 \frac{\zeta^{\prime}}{\zeta}(2)+2 \gamma_{\mathrm{e}}\right)\right)
$$

and $\Lambda_{0}(a)$ as in Lemma 4.2. Suppose that $-1 / 2<a<0$. By definition

$\Phi(-a, x)=\Lambda_{0}(-a) \log x+\Lambda_{1}(-a) \eta_{1}(-a, x)+\Lambda_{2}(-a) \eta_{0}(-a, x)+\frac{\Lambda_{0}(a)}{1+2 a} \eta_{1}(-2 a, x)$.

Observe that $\eta_{0}(-a, x)=a^{-2} \varphi_{0}^{*}(-a \log x)$, where $\varphi_{0}^{*}(y):=1-y e^{-y}-e^{-y}$ is monotone increasing and bounded for $y \geq 0$. Then

$$
\left|\frac{\Lambda_{2}(-a)}{\Lambda_{0}(-a) \log x} \sum_{n=0}^{N} 2^{(2 a-1) n} \eta_{0}\left(-a, 2^{-n} x\right)\right| \ll|a \log x|^{-1} \varphi_{0}^{*}(-a \log x) \ll|a \log x|^{-1}
$$


as $|a \log x|^{-1} \rightarrow 0$. Similarly

$$
\left|\frac{1}{\Lambda_{0}(-a) \log x} \sum_{n=0}^{N} 2^{(2 a-1) n}\left(\Lambda_{1}(-a) \eta_{1}\left(-a, 2^{-n} x\right)+\frac{\Lambda_{0}(a)}{1+2 a} \eta_{1}\left(-2 a, 2^{-n} x\right)\right)\right|
$$

is also $\ll|a \log x|^{-1}$. Combining these estimates with (4.8) we obtain the asymptotic formula for $-1 / 2<a<0$. In the case $a \leq-1 / 2$, the desired formula immediately follows from $\Phi(-a, x)=\Lambda_{0}(-a) \log x$. Thus the lemma is verified.

4.4. Proof of Lemma 4.3. We need the following:

LEMMA 4.6. As $|a|+(\log x)^{-1} \rightarrow 0, \Lambda(a, x) \asymp l_{3}(a, x)(>0)$.

PROOF. Recall expression (4.1) with $l_{3}(a, x)=3 a^{-3} \varphi_{3}(a \log x)$ and $l_{2}^{1}(a, x)=$ $a^{-2} \varphi_{2}^{1}(a \log x)$, where $\varphi_{3}(y)=1-2 y e^{-y}-e^{-2 y}$ and $\varphi_{2}^{1}(y):=e^{-y}\left(1-y-e^{-y}\right)$. These functions satisfy $\left|\varphi_{3}(y)\right| \asymp|y|^{3}$ and $\left|\varphi_{2}^{1}(y)\right| \asymp y^{2}$ for $|y| \ll 1$. If $|a \log x| \ll 1$, then $l_{2}^{1}(a, x) / l_{3}(a, x) \ll\left|a \varphi_{2}^{1}(a \log x) / \varphi_{3}(a \log x)\right| \asymp(\log x)^{-1}$, and if $|a \log x| \gg 1$, then $l_{2}^{1}(a, x) / l_{3}(a, x) \ll|a|$. Hence we have $l_{2}^{1}(a, x) / l_{3}(a, x) \ll|a|+(\log x)^{-1}$. Similarly $l_{3}(a, x)^{-1}\left(\left|l_{2}^{2}(a, x)\right|+\left|l_{1}^{1}(a, x)\right|+\left|l_{1}^{2}(a, x)\right|+x^{-a} \log x+x^{-2 a}+1\right) \ll|a|+(\log x)^{-1}$. These estimates imply the lemma.

LEMMA 4.7. Let $0<\beta \leq 1$. Then, $\Lambda(a, x) \gg\left(1+x^{-2 a}\right)(\log x)^{3 \beta}$ if $|a| \ll$ $(\log x)^{-\beta}$. Furthermore, as $|a \log x|^{-1} \rightarrow 0, \Lambda(a, x) \asymp \Lambda_{0}(a)$ if $a>0$ (respectively, $\Lambda(a, x) \asymp(1-2 a)^{-1} \Lambda_{0}(-a) x^{-2 a}$ if $\left.a<0\right)$.

PROOF. If $0 \leq a \ll(\log x)^{-\beta}$, then, by Lemma 4.6,

$$
\Lambda(a, x) \asymp l_{3}(a, x)=3\left|a^{-3} \varphi_{3}(a \log x)\right| \gg \min \left\{(\log x)^{3}, a^{-3}\right\} \gg(\log x)^{3 \beta},
$$

since $\left|\varphi_{3}(y)\right|=\left|1-2 y e^{-y}-e^{-2 y}\right| \gg \min \left\{y^{3}, 1\right\}$ for $y \geq 0$. Using $\left|\varphi_{3}(y)\right| \gg$ $e^{-2 y} \min \left\{|y|^{3}, 1\right\}$ for $y \leq 0$, we have $\Lambda(a, x) \gg x^{-2 a}(\log x)^{3 \beta}$ if $0 \leq-a \ll(\log x)^{-\beta}$. If $0<a<1 / 2$ (respectively, $-1 / 2<a<0)$ and if $(a \log x)^{-1}$ is sufficiently small, we obtain from (4.9) that $\Lambda(a, x) \asymp \Lambda_{0}(a)$ (respectively, $\asymp(1-2 a)^{-1} \Lambda_{0}(-a) x^{-2 a}$ ), since $(1+|a| \log x) x^{-|a|} \ll \exp (-|a| \log x / 2)$. If $a \geq 1 / 2$ (respectively, $\left.a \leq-1 / 2\right)$ the same estimate immediately follows.

To prove Lemma 4.3 we first suppose that $|a|+(\log x)^{-1}$ is sufficiently small. Since $2^{-N} x \asymp x^{1 / 4},\left(\log \left(2^{-n} x\right)\right)^{-1} \asymp(\log x)^{-1}$ for $n \leq N$. Using Lemma 4.6 and observing that $\varphi_{3}(y)$ is monotone increasing for $y \in \mathbf{R}$, we have

$$
|\Lambda|_{N}(a, x)=\sum_{n=0}^{N} 2^{-n}\left|\Lambda\left(a, 2^{-n} x\right)\right| \ll \sum_{n=0}^{N} 2^{-n} l_{3}\left(a, 2^{-n} x\right) \ll l_{3}(a, x) .
$$


By Lemma 4.1, $\Phi_{N}(a, x) \asymp a^{-4} \psi(a, x)=a^{-4} h_{0}(a \log x)$. Estimates (4.5) and (4.6) yield $\varphi_{3}(y) / h_{0}(y) \ll 1+|y|^{-1}$ uniformly in $y \in \mathbf{R}$. Hence

$$
|\Lambda|_{N}(a, x) / \Phi_{N}(a, x) \ll\left|a \varphi_{3}(a \log x) / h_{0}(a \log x)\right| \ll|a|+(\log x)^{-1}
$$

as $|a|+(\log x)^{-1} \rightarrow 0$. If $a<0$, then, by Lemma 4.7, $\Lambda(a, x) \ll \Lambda_{0}(-a) x^{-2 a}$ as $|a \log x|^{-1} \rightarrow 0$. Hence by Lemma 4.2, $|\Lambda|_{N}(a, x) \ll \Lambda_{0}(-a) x^{-2 a} \ll \widetilde{\Phi}_{N}(a, x)(\log x)^{-1}$. Thus we obtain Lemma 4.3.

4.5. Proof of Lemma 4.4. Lemma 4.4 immediately follows from Lemma 4.7 and the following with $c_{0}>0$ :

LEMMA 4.8. (1) Suppose that $a \geq 0$. For $\mu-1 \geq c_{0}$ (respectively, $\mu-1 \leq-c_{0}$ ),

$$
\int_{x}^{\infty} \xi^{-\mu} \Lambda(a, \xi) d \xi\left(\text { respectively, } \int_{1}^{x} \xi^{-\mu} \Lambda(a, \xi) d \xi\right) \ll \frac{x^{1-\mu} \Lambda(a, x)}{|\mu-1|},
$$

the implied constant depending on $c_{0}$ only.

(2) Suppose that $a<0$. For $\mu+2 a-1 \geq c_{0}$ (respectively, $\mu-1 \leq-c_{0}$ ), the integral above is $\ll|\mu-1|^{-1}(|a|+1) x^{1-\mu} \Lambda(a, x)$, the implied constant depending on $c_{0}$ only.

Indeed, for example, in the case $a<0$, denoting $\Sigma(a, x):=\sum_{n \leq x} \sigma_{-a}(n)^{2}$ and using Proposition 3.1, we derive

$$
\begin{gathered}
\sum_{n \leq \tau} \frac{\sigma_{-a}(n)^{2}}{n}=\tau^{-1} \Sigma(a, \tau)+\int_{1}^{\tau} \xi^{-2} \Sigma(a, \xi) d \xi \\
=\Lambda(a, \tau)+\int_{1}^{\tau} \xi^{-1} \Lambda(a, \xi) d \xi+O\left(\tau^{-2 a-1 / 2+\varepsilon}+1\right) \\
=\Phi(a, \tau)+O((1+|a|) \Lambda(a, \tau))=\Phi(a, \tau)+O\left(\Lambda^{*}(a, \tau)\right)
\end{gathered}
$$

with the absolute implied constants, and if $\mu+2 a-1 \geq c_{0}$,

$$
\begin{gathered}
\sum_{n>\tau} \frac{\sigma_{-a}(n)^{2}}{n^{\mu}}=\tau^{-\mu} \Sigma(a, \tau)+\mu \int_{\tau}^{\infty} \xi^{-\mu-1} \Sigma(a, \xi) d \xi \\
\ll \tau^{1-\mu} \Lambda(a, \tau)+\mu \int_{\tau}^{\infty} \xi^{-\mu} \Lambda(a, \xi) d \xi+\frac{\mu \tau^{-2 a+1 / 2-\mu+\varepsilon}}{|2 a+\mu|+1} \\
\ll(1+|a|) \tau^{1-\mu}\left(\Lambda(a, \tau)+O\left(\tau^{-2 a}\right)\right) \ll(1+|a|)^{2} \tau^{1-\mu} \Lambda(a, \tau) \ll \tau^{1-\mu} \Lambda^{*}(a, \tau)
\end{gathered}
$$

with the implied constants depending on $c_{0}$ only.

\subsubsection{Proof of Lemma 4.8 in the case $a \geq 0$}

Suppose that $\mu-1 \geq c_{0}$. We have

$$
\int_{x}^{\infty} \xi^{-\mu} \Lambda(a, \xi) d \xi=\frac{x^{1-\mu} \Lambda(a, x)}{\mu-1}+\frac{J(a, x)}{\mu-1}, \quad J(a, x):=\int_{x}^{\infty} \xi^{1-\mu} \Lambda_{\xi}(a, \xi) d \xi
$$


where $\Lambda_{\xi}(a, \xi):=(\partial / \partial \xi) \Lambda(a, \xi)$, provided that $J(a, x)$ converges. If $a \geq 1 / 2$, then $\Lambda_{\xi}(a, \xi) \equiv 0$, and hence the conclusion immediately follows. For $0 \leq a<1 / 2$, by (4.9)

$$
\xi \Lambda_{\xi}(a, \xi)=\Lambda_{2}(a) \xi^{-a}(1-a \log \xi)-a \Lambda_{1}(a) \xi^{-a}-\frac{2 a \Lambda_{0}(-a)}{1-2 a} \xi^{-2 a} \ll \Lambda_{0}(a),
$$

which is checked by using $a x^{-a} \log x \ll 1$ and $\left(\left|\Lambda_{2}(a)\right|+\left|\Lambda_{1}(a)\right|\right) / \Lambda_{0}(a) \ll a$. This gives $J(a, x) \ll \Lambda_{0}(a) x^{1-\mu}$ with the implied constant depending on $c_{0}$ only. Then, by Lemma 4.7 , we obtain the conclusion, say for $(\log x)^{-5 / 6} \ll a<1 / 2$. To complete the proof for $a \geq 0$ it is sufficient to show the estimate for $0 \leq a \ll(\log x)^{-2 / 3}$. To do so we recall (4.1). Under this condition, $l_{3}^{\prime}(a, x) \ll a^{-2} x^{-a-1}\left|-1+a \log x+x^{-a}\right| \ll x^{-a-1}(\log x)^{2}$, and similarly $\left|\left(l_{i}^{j}\right)^{\prime}(a, x)\right| \ll x^{-a-1}(\log x)^{2}$. Hence

$$
J(a, x) \ll \int_{x}^{\infty} \xi^{-a-\mu}(\log \xi)^{2} d \xi \ll x^{1-\mu-a}(\log x)^{2} .
$$

By Lemma 4.7 with $\beta=2 / 3, J(a, x) \ll x^{1-\mu}(\log x)^{2} \ll x^{1-\mu} \Lambda(a, x)$. Thus we obtain the lemma for $\mu-1 \geq c_{0}$. The integral with $\mu-1 \leq-c_{0}$ may be treated similarly.

\subsubsection{Proof of Lemma 4.8 in the case $a<0$}

If $a \leq-1 / 2$, the conclusion is immediately obtained. For $-1 / 2<a<0$ and for $\mu+2 a-1 \geq c_{0}$, write $\xi^{1-\mu} \Lambda_{\xi}(a, \xi)=\xi^{-\mu-2 a} \cdot \xi^{1+2 a} \Lambda_{\xi}(a, \xi) \ll \Lambda_{0}(-a) \xi^{-\mu-2 a}$. If $(\log x)^{-5 / 6} \ll-a<1 / 2$, we have $J(a, x) \ll x^{1-\mu} \Lambda(a, x)$. Furthermore, if $0<-a \ll$ $(\log x)^{-2 / 3}$, observing $l_{3}^{\prime}(a, x) \ll a^{-2} x^{-2 a-1}\left|1+a x^{a} \log x-x^{a}\right| \ll x^{-2 a-1}(\log x)^{2}$ and so on, we deduce that $J(a, x) \ll x^{1-\mu} \Lambda(a, x)$. The remaining case $\mu-1 \leq-c_{0}$ is similarly treated.

4.6. Proof of Lemma 4.5. Set $U_{a}(x):=\sum_{n \leq x} \sigma_{a}(n)$. If $c_{0} \leq 1-\sigma<1$,

$$
\sum_{n \leq \tau} \frac{\sigma_{a}(n)}{n^{\sigma}} \ll \tau^{-\sigma} U_{a}(\tau)+\int_{1}^{\tau} \xi^{-\sigma-1} U_{a}(\xi) d \xi \ll(1+|a|)\left|\frac{1-\tau^{a}}{a}\right| \frac{\tau^{1-\sigma}}{c_{0}},
$$

since, by Proposition 3.2, $(1+|a|)^{-1} \xi^{-\sigma-1} U_{a}(\xi) \ll \xi^{-\sigma}\left|\left(1-\xi^{a}\right) / a\right| \leq \xi^{-\sigma}\left|\left(1-\tau^{a}\right) / a\right|$ for $1 \leq \xi \leq \tau$ and for $a \in \mathbf{R}$. If $\sigma \geq 1+\max \{0, a\}+c_{0}$,

$$
\begin{gathered}
\sum_{n>\tau} \frac{\sigma_{a}(n)}{n^{\sigma}} \ll \tau^{-\sigma} U_{a}(\tau)+\sigma \int_{\tau}^{\infty} \xi^{-\sigma-1} U_{a}(\xi) d \xi \\
\ll \tau^{-\sigma} U_{a}(\tau)+\frac{(1+|a|) \sigma}{|a|} \int_{\tau}^{\infty}\left|\xi^{-\sigma}-\xi^{-\sigma+a}\right| d \xi \ll(1+|a|) \frac{\tau^{1-\sigma}}{c_{0}}\left(\left|\frac{1-\tau^{a}}{a}\right|+\frac{\tau^{a}}{c_{0}}\right) .
\end{gathered}
$$

Thus we obtain Lemma 4.5. 


\section{Integral related to the proofs of the main results}

For $\tau \gg 1$, consider the integral

$$
I(\tau / 2, \tau):=\int_{\tau / 2}^{\tau}\left|\zeta\left(\frac{1}{2}+i t\right) \zeta\left(\frac{1}{2}+a+i t\right)\right|^{2} d t
$$

In this section we show the following, which will be used in the proofs of our main results.

PROPOSITION 5.1. If $-\tau^{1 / 4} \leq a \leq 1 / 16$, then

$$
I(\tau / 2, \tau)=\frac{\tau}{2}\left(\Phi(a, \tau)+(2 \pi)^{2 a} \lambda(a) \tau^{-2 a} \Phi(-a, \tau)\left(1+O\left(\tau^{-1 / 4}\right)\right)+R_{0}(a, \tau)\right)
$$

with $R_{0}(a, \tau) \ll\left(|a|^{2|a|}+1\right)\left(\Lambda(a, \tau)+\left|\left(1-\tau^{-a}\right) / a\right|^{2}\right)$ and $\lambda(a):=2\left(1-2^{2 a-1}\right) /(1-2 a)$. Here the implied constants are absolute.

5.1. Approximate formula. For $\zeta(s) \zeta(s+a)$ we give an approximate formula by the reflection principle. Let $a$ satisfy $-\tau^{1 / 4} \leq a \leq 1 / 16$. Suppose that $\tau / 2 \leq t \leq \tau$ and that $|\sigma-1 / 2|<1 / 4$. Then

$$
\sum_{n=1}^{\infty} \sigma_{-a}(n) e^{-n / \tau} n^{-s}=\frac{1}{2 \pi i} \int_{(2+|a|)} \zeta(s+w) \zeta(s+a+w) \Gamma(w) \tau^{w} d w,
$$

where $s=\sigma+i t, w=u+i v$, and the symbol $(\alpha)$ denotes the vertical line $w=\alpha+i v$, $-\infty<v<\infty$, since $\zeta(s) \zeta(s+a)=\sum_{n=1}^{\infty} \sigma_{-a}(n) n^{-s}$ for $\operatorname{Re}(s)>\max \{1,1-a\}$. If $a \neq 0$, the poles of the integrand in the strip $-3 / 4<u<2+|a|$ are $w=0,1-s$, $1-s-a$, whose residues are $R_{0}:=\zeta(s) \zeta(s+a), R_{1-s}:=\zeta(1+a) \Gamma(1-s) \tau^{1-s}$ and $R_{1-s-a}:=\zeta(1-a) \Gamma(1-s-a) \tau^{1-s-a}$, respectively. Note that

$$
R_{1-s}+R_{1-s-a}=\Gamma(1-s) \tau^{1-s}(\zeta(1+a)+\zeta(1-a)-a \zeta(1-a) g(-a, s))
$$

with $g(z, s)=z^{-1}\left(\Gamma(1-s+z) \tau^{z} / \Gamma(1-s)-1\right)$, and that

$$
|\Gamma(\sigma+i t)|=\sqrt{2 \pi}|t|^{\sigma-1 / 2} e^{-(\pi / 2)|t|}\left(1+O\left(|t|^{-1 / 2}\right)\right)
$$

for $|\sigma| \ll|t|^{1 / 4},|t| \geq 1$, which may be checked by using Stirling's formula. Since $g(z, s)$ is holomorphic around $z=0$, by the maximal modulus principle together with (5.2), we have $g(-a, s) \ll \max _{|z|=1}\left|\Gamma(1-s+z) \tau^{z} / \Gamma(1-s)\right| \ll \tau^{2}$ for $|a| \leq 1 / 16$, so that $R_{1-s}+$ $R_{1-s-a} \ll e^{-t}$. For $-\tau^{1 / 4} \leq a \leq-1 / 16$, we have $R_{1-s-a} \ll|\Gamma(1-s-a)| \tau^{1-\sigma-a} \ll$ $\tau^{1-2 a} e^{-(\pi / 2) t} \ll e^{-t}$, and $R_{1-s} \ll|\zeta(-a) \chi(1+a) \Gamma(1-s)| \tau^{1-\sigma} \ll \Gamma(-a)|\Gamma(1-s)| \tau \ll$ $e^{-t}$, where $\chi(s)=2^{s} \pi^{s-1} \sin (\pi s / 2) \Gamma(1-s)$. In (5.1) we shift the path of integration to $(-3 / 4)$ and use the functional equation for $\zeta(s)$ to find

$$
\frac{1}{2 \pi i} \int_{(-3 / 4)} \chi(s+w) \chi(s+w+a) \Gamma(w) \tau^{w}\left(\sum_{n=1}^{\infty} \frac{\sigma_{a}(n)}{n^{1-s-w}}\right) d w
$$




$$
=\sum_{n=1}^{\infty} \sigma_{-a}(n) e^{-n / \tau} n^{-s}-\zeta(s) \zeta(s+a)+O\left(e^{-\tau / 2}\right)
$$

for $|\sigma-1 / 2|<1 / 4$ and for $-\tau^{1 / 4} \leq a \leq 1 / 16$. The sum in the integrand above converges. Split the sum into two parts $\sum_{n<\tau}$ and $\sum_{n>\tau}$. For the integral related to $\sum_{n<\tau}$, shift the path of integration to the line $(1 / 4)$. Between $(-3 / 4)$ and $(1 / 4)$, the integrand possesses a pole only at $w=0$, whose residue is $\chi(s) \chi(s+a) \sum_{n \leq \tau} \sigma_{a}(n) n^{s-1}$. Consequently

$$
\zeta(s) \zeta(s+a)=S_{1}(a, s)+S_{2}(a, s)+\sum_{j=1}^{4} F_{j}(a, s)+O\left(e^{-\tau / 2}\right)
$$

for $|\sigma-1 / 2| \leq 1 / 8, \tau / 2 \leq t \leq \tau,-\tau^{1 / 4} \leq a \leq 1 / 16$, where

$$
\begin{aligned}
& S_{1}(a, s):=\sum_{n \leq \tau} \sigma_{-a}(n) n^{-s}, \quad S_{2}(a, s):=\chi(s) \chi(s+a) \sum_{n \leq \tau} \sigma_{a}(n) n^{s-1}, \\
& F_{1}(a, s):=\sum_{n \leq \tau} \sigma_{-a}(n) n^{-s}\left(e^{-n / \tau}-1\right), \quad F_{2}(a, s):=\sum_{n>\tau} \sigma_{-a}(n) n^{-s} e^{-n / \tau}, \\
& F_{3}(a, s):=-\frac{1}{2 \pi i} \int_{(-3 / 4)} \chi(s+w) \chi(s+w+a) \Gamma(w) \tau^{w}\left(\sum_{n>\tau} \frac{\sigma_{a}(n)}{n^{1-s-w}}\right) d w, \\
& F_{4}(a, s):=-\frac{1}{2 \pi i} \int_{(1 / 4)} \chi(s+w) \chi(s+w+a) \Gamma(w) \tau^{w}\left(\sum_{n \leq \tau} \frac{\sigma_{a}(n)}{n^{1-s-w}}\right) d w .
\end{aligned}
$$

5.2. Expression of the integral. Suppose that $|\sigma-1 / 2| \leq 1 / 8$. By (5.2), for $\tau / 2 \leq$ $t \leq \tau$, for $a \leq 1 / 16$ and for each fixed $u \ll 1$,

$$
\begin{aligned}
\chi(s+ & w) \chi(s+w+a) \Gamma(w) \tau^{w} \\
& \ll(2 \pi)^{2 u+a}(1+|v+t|)^{1-2 \sigma-2 u-a}(1+|v|)^{u-1 / 2} e^{-(\pi / 2)|v|} \tau^{u} \\
& \ll \tau^{u} t^{1-2 \sigma-2 u-a}\left(1+\frac{|v|}{t}\right)^{1-2 \sigma-2 u-a}(1+|v|)^{u-1 / 2} e^{-(\pi / 2)|v|} \\
& \ll \tau^{1-2 \sigma-u-a}(1+|v|)^{1 / 2-2 \sigma-u-a} e^{-(\pi / 2)|v|} .
\end{aligned}
$$

Along the line $u=-3 / 4, \sum_{n>\tau} \sigma_{a}(n) / n^{1-\sigma-u} \ll \sum_{n>\tau}\left(1+n^{a}\right) d(n) / n^{7 / 4-\sigma} \ll$ $\tau^{\sigma-3 / 4+\varepsilon}\left(1+\tau^{a}\right)$ if $a \leq 1 / 16$. Hence $F_{3}(a, s) \ll \Gamma(|a|+2) \tau^{1-\sigma+\varepsilon}\left(1+\tau^{-a}\right) \ll$ $\exp \left(2 \tau^{1 / 4+\varepsilon}\right)$ for $|\sigma-1 / 2| \leq 1 / 8$ and for $-\tau^{1 / 4} \leq a \leq 1 / 16$. Furthermore $F_{2}(a, s) \ll$ $(|a|+1)^{|a|} \sum_{n>\tau} \sigma_{-a}(n) n^{-\sigma}(\tau / n)^{|a|+1} \ll \tau^{2|a|} \sum_{n>\tau}\left(1+n^{-a}\right) d(n) n^{-\sigma-|a|-1} \ll \tau^{2|a|} \ll$ $\exp \left(2 \tau^{1 / 4+\varepsilon}\right)$, since $e^{-n / \tau} \ll((|a|+1) / e)^{|a|+1}(\tau / n)^{|a|+1}$. Similarly, $S_{j}(a, s), F_{j^{\prime}}(a, s) \ll$ $\exp \left(2 \tau^{1 / 4+\varepsilon}\right)\left(j=1,2 ; j^{\prime}=1,4\right)$. Using formula (5.3) together with these estimates, we 
derive

$$
I(\tau / 2, \tau)=\sum_{k=1}^{6} J_{k}+O\left(\tau^{-1}\right)
$$

for $-\tau^{1 / 4} \leq a \leq 1 / 16$, where

$$
\begin{aligned}
& J_{1}:=\int_{\tau / 2}^{\tau}\left|S_{1}(a, 1 / 2+i t)\right|^{2} d t+\int_{\tau / 2}^{\tau}\left|S_{2}(a, 1 / 2+i t)\right|^{2} d t, \\
& J_{2}:=\sum_{j=1}^{4} \int_{\tau / 2}^{\tau}\left|F_{j}(a, 1 / 2+i t)\right|^{2} d t, \\
& J_{3}:=\int_{\tau / 2}^{\tau}\left(S_{1}(a, 1 / 2+i t) S_{2}(a, 1 / 2-i t)+S_{1}(a, 1 / 2-i t) S_{2}(a, 1 / 2+i t)\right) d t, \\
& J_{4}=\bar{J}_{5}:=\sum_{j=1}^{4} \int_{\tau / 2}^{\tau}\left(S_{1}(a, 1 / 2+i t)+S_{2}(a, 1 / 2+i t)\right)(-1)^{\iota(j)} F_{j}(a, 1 / 2-i t) d t, \\
& J_{6}:=\sum_{\substack{1 \leq j \leq 4 \\
1 \leq j^{\prime} \leq 4 \\
j \neq j^{\prime}}}^{\tau} \int_{\tau / 2}^{\tau}(-1)^{\iota\left(j^{\prime}\right)} F_{j}(a, 1 / 2+i t) F_{j^{\prime}}(a, 1 / 2-i t) d t
\end{aligned}
$$

with $\iota(1)=\iota(2)=0, \iota(3)=\iota(4)=1$. Evaluation of these integrals basically depends on the identity of Montgomery and Vaughan ([9], [6, §§5.1, 5.2]):

PROPOSITION 5.2. Let $\gamma_{1}, \ldots, \gamma_{N}$ be arbitrary complex numbers. Then

$$
\int_{0}^{\tau}\left|\sum_{n \leq N} \gamma_{n} n^{i t}\right|^{2} d t=\tau \sum_{n \leq N}\left|\gamma_{n}\right|^{2}+O\left(\sum_{n \leq N} n\left|\gamma_{n}\right|^{2}\right) .
$$

This remains valid for $N=\infty$ as well, provided that the series on the right-hand side converge.

Furthermore we need

LEMMA 5.3. For $a \leq 1 / 16$ we have $\tau^{-2 a} \Lambda^{*}(-a, \tau) \asymp(|a|+1) \Lambda^{*}(a, \tau)$.

Proof. For $|a| \ll(\log \tau)^{-1 / 3}$, by Lemma 4.6,

$$
\begin{gathered}
\Lambda(a, \tau) \asymp l_{3}(a, \tau) \asymp\left|a^{-3}\left(1-2 \tau^{-a} \cdot a \log \tau-\tau^{-2 a}\right)\right| \\
=\tau^{-2 a}\left|a^{-3}\left(1-2 \tau^{a}(-a \log \tau)-\tau^{2 a}\right)\right| \asymp \tau^{-2 a} l_{3}(-a, \tau) \asymp \tau^{-2 a} \Lambda(-a, \tau) .
\end{gathered}
$$

For $(\log \tau)^{-1 / 2} \ll|a|$, by Lemma 4.7, we have $\Lambda(a, \tau) \asymp \Lambda_{0}(a)$ if $a>0$, and $\Lambda(a, \tau) \asymp$ $(1-2 a)^{-1} \Lambda_{0}(-a) \tau^{-2 a}$ if $a<0$. From these facts and the definition of $\Lambda^{*}(a, \tau)$ the lemma immediately follows. 
In the remaining part of this section we give an asymptotic expression for $J_{1}$ and estimates for $J_{j}(2 \leq j \leq 6)$, from which Proposition 5.1 immediately follows.

5.3. Main terms. Since $\left|S_{1}(a, 1 / 2+i t)\right|^{2}=\left|\sum_{n \leq \tau} \sigma_{-a}(n) n^{-1 / 2-i t}\right|^{2}$, we apply Lemma 4.4 and Proposition 5.2 to obtain

$$
\begin{gathered}
\int_{\tau / 2}^{\tau}\left|S_{1}(a, 1 / 2+i t)\right|^{2} d t=\frac{\tau}{2} \sum_{n \leq \tau} \frac{\sigma_{-a}(n)^{2}}{n}+O\left(\sum_{n \leq \tau} \sigma_{-a}(n)^{2}\right) \\
=\frac{\tau}{2}\left(\Phi(a, \tau)+O\left(\Lambda^{*}(a, \tau)\right)\right) .
\end{gathered}
$$

By (5.2), $|\chi(1 / 2+i t) \chi(1 / 2+a+i t)|=(2 \pi / t)^{a}\left(1+O\left(t^{-1 / 2}\right)\right)$ for $-\tau^{1 / 4} \leq a \leq 1 / 16$ and for $\tau / 2 \leq t \leq \tau$. Hence

$$
I:=\int_{\tau / 2}^{\tau}\left|S_{2}(a, 1 / 2+i t)\right|^{2} d t=(2 \pi)^{2 a}\left(\int_{\tau / 2}^{\tau} t^{-2 a} F^{\prime}(t) d t+O\left(\int_{\tau / 2}^{\tau} t^{-2 a-1 / 2} F^{\prime}(t) d t\right)\right)
$$

with

$$
F(x):=\int_{\tau / 2}^{x}\left|\sum_{n \leq \tau} \frac{\sigma_{a}(n)}{n^{1 / 2-i t}}\right|^{2} d t
$$

Integrating by parts and observing

$$
\int_{\tau / 2}^{\tau} t^{-2 a-1 / 2} F^{\prime}(t) d t \ll \tau^{-2 a-1 / 2} \int_{\tau / 2}^{\tau} F^{\prime}(t) d t=\tau^{-2 a-1 / 2} F(\tau),
$$

we find

$$
I=(2 \pi)^{2 a}\left(\tau^{-2 a} F(\tau)\left(1+O\left(\tau^{-1 / 2}\right)\right)+2 a \int_{\tau / 2}^{\tau} t^{-2 a-1} F(t) d t\right) .
$$

Since, by Lemma 4.4 and Proposition 5.2, $F(x)=(x-\tau / 2) \Phi(-a, \tau)+O\left(\tau \Lambda^{*}(-a, \tau)\right)$ for $\tau / 2 \leq x \leq \tau$, it follows that

$$
2 a \int_{\tau / 2}^{\tau} t^{-2 a-1} F(t) d t=\frac{2 a+1-2^{2 a}}{2(1-2 a)} \tau^{1-2 a} \Phi(-a, \tau)+O\left(\tau^{1-2 a} \Lambda^{*}(-a, \tau)\right),
$$

and hence, by Lemma 5.3,

$$
I=(2 \pi)^{2 a} \tau\left(\frac{1-2^{2 a-1}}{1-2 a} \tau^{-2 a} \Phi(-a, \tau)\left(1+O\left((|a|+1) \tau^{-1 / 2}\right)\right)+O\left((|a|+1) \Lambda^{*}(a, \tau)\right)\right) .
$$

Combining this with (5.6), we have, for $-\tau^{1 / 4} \leq a \leq 1 / 16$,

(5.7) $J_{1}=\frac{\tau}{2}\left(\Phi(a, \tau)+(2 \pi)^{2 a} \lambda(a) \tau^{-2 a} \Phi(-a, \tau)\left(1+O\left(\tau^{-1 / 4}\right)\right)+O\left(\left(1+a^{2}\right) \Lambda(a, \tau)\right)\right)$. 
5.4. Evaluation of $J_{2}$ and $J_{6}$. Along the line $w=-3 / 4+i v,-\infty<v<\infty$, $\left|\chi(w+1 / 2+i t) \chi(w+1 / 2+a+i t) \Gamma(w) \tau^{w}\right| \ll \tau^{3 / 4-a}(1+|v|)^{1 / 4-a} e^{-\pi|v| / 2}$ (cf. (5.4)), and hence

$$
\begin{aligned}
& \left|F_{3}(a, 1 / 2+i t)\right|^{2} \ll \int_{-\infty}^{\infty}\left|\sum_{n>\tau} \frac{\sigma_{a}(n) n^{i v}}{n^{5 / 4-i t}}\right|^{2} e^{-\pi|v| / 2} d v \\
& \quad \times \int_{(-3 / 4)}\left|\chi(w+1 / 2+i t) \chi(w+1 / 2+a+i t) \Gamma(w) \tau^{w} e^{\pi|v| / 4}\right|^{2}|d w| \\
& \ll \Gamma(2|a|+2) \tau^{3 / 2-2 a} \int_{-\infty}^{\infty}\left|\sum_{n>\tau} \frac{\sigma_{a}(n) n^{i v}}{n^{5 / 4-i t}}\right|^{2} e^{-\pi|v| / 2} d v .
\end{aligned}
$$

By Lemma 4.4,

$$
\tau \sum_{n>\tau}\left|\frac{\sigma_{a}(n) n^{i v}}{n^{5 / 4}}\right|^{2}+\sum_{n>\tau} n\left|\frac{\sigma_{a}(n) n^{i v}}{n^{5 / 4}}\right|^{2} \ll \tau^{-1 / 2} \Lambda^{*}(-a, \tau) .
$$

This gives

$$
\begin{gathered}
\int_{\tau / 2}^{\tau} \frac{\left|F_{3}(a, 1 / 2+i t)\right|^{2}}{\Gamma(2|a|+2)} d t \ll \tau^{3 / 2-2 a} \int_{-\infty}^{\infty}\left(\int_{\tau / 2}^{\tau}\left|\sum_{n>\tau} \frac{\sigma_{a}(n) n^{i v}}{n^{5 / 4-i t}}\right|^{2} d t\right) e^{-\pi|v| / 2} d v \\
\ll \tau^{3 / 2-2 a} \cdot \tau^{-1 / 2} \Lambda^{*}(-a, \tau) \ll\left(1+|a|^{3}\right) \tau \Lambda(a, \tau) .
\end{gathered}
$$

Similarly we have

$$
\begin{gathered}
\int_{\tau / 2}^{\tau} \frac{\left|F_{4}(a, 1 / 2+i t)\right|^{2}}{\Gamma(2|a|+2)} d t \ll \tau^{-1 / 2-2 a} \int_{-\infty}^{\infty}\left(\int_{\tau / 2}^{\tau}\left|\sum_{n \leq \tau} \frac{\sigma_{a}(n) n^{i v}}{n^{1 / 4-i t}}\right|^{2} d t\right) e^{-\pi|v| / 2} d v \\
\ll \tau^{-1 / 2-2 a} \cdot \tau^{3 / 2} \Lambda^{*}(-a, \tau) \ll\left(1+|a|^{3}\right) \tau \Lambda(a, \tau) .
\end{gathered}
$$

Since $e^{-2 n / \tau} \ll((|a|+1) / e)^{2|a|+2}(\tau / n)^{2|a|+2} \ll\left(1+|a|^{2|a|-2}\right)(\tau / n)^{2|a|+2}$ for $n \geq \tau$,

$$
\begin{gathered}
\int_{\tau / 2}^{\tau}\left|F_{2}(a, 1 / 2+i t)\right|^{2} d t \ll \tau \sum_{n>\tau} \sigma_{-a}(n)^{2} n^{-1} e^{-2 n / \tau}+\sum_{n>\tau} \sigma_{-a}(n)^{2} e^{-2 n / \tau} \\
\ll\left(1+|a|^{2|a|-2}\right)\left(\tau^{2|a|+3} \sum_{n>\tau} \sigma_{-a}(n)^{2} n^{-2|a|-3}+\tau^{2|a|+2} \sum_{n>\tau} \sigma_{-a}(n)^{2} n^{-2|a|-2}\right) \\
\ll\left(1+|a|^{2|a|-2}\right) \tau \Lambda^{*}(a, \tau) \ll\left(1+|a|^{2|a|}\right) \tau \Lambda(a, \tau) .
\end{gathered}
$$

The integral corresponding to $F_{1}(a, 1 / 2+i t)$ may be treated in a similar way by using $e^{-n / \tau}-$ $1 \ll n / \tau$ for $n \leq \tau$. Thus we obtain $J_{2} \ll\left(1+|a|^{2|a|}\right) \tau \Lambda(a, \tau)$. The inequalities

$$
\int_{\tau / 2}^{\tau}\left|F_{j}(a, 1 / 2+i t) F_{j^{\prime}}(a, 1 / 2-i t)\right| d t
$$




$$
\leq\left(\int_{\tau / 2}^{\tau}\left|F_{j}(a, 1 / 2+i t)\right|^{2} d t \int_{\tau / 2}^{\tau}\left|F_{j^{\prime}}(a, 1 / 2+i t)\right|^{2} d t\right)^{1 / 2},
$$

for $1 \leq j \leq 4,1 \leq j^{\prime} \leq 4, j \neq j^{\prime}$ yield $J_{6} \ll\left(1+|a|^{2|a|}\right) \tau \Lambda(a, \tau)$.

5.5. Evaluation of $J_{3}$. Let $G(\sigma)$ denote the integral

$$
\int_{\tau / 2}^{\tau} S_{1}(a, s) S_{2}(a, 1-s) d t=\int_{\tau / 2}^{\tau} \chi(1-s) \chi(1-s+a)\left(\sum_{n \leq \tau} \frac{\sigma_{-a}(n)}{n^{s}}\right)\left(\sum_{n \leq \tau} \frac{\sigma_{a}(n)}{n^{s}}\right) d t .
$$

Then $J_{3}$ is written in the form $J_{3}=G(1 / 2)+\overline{G(1 / 2)}$. To evaluate $G(1 / 2)$ shift the segment of integration $[1 / 2+i \tau / 2,1 / 2+i \tau]$ to $[3 / 8+i \tau / 2,3 / 8+i \tau]$. By Lemma 4.5 together with $\chi(1-s) \chi(1-s+a) \ll t^{2 \sigma-1-a}$, the integrand along the horizontal segment $[3 / 8+$ $i \tau / 2,1 / 2+i \tau / 2]$ or $[3 / 8+i \tau, 1 / 2+i \tau]$ is $\ll\left(1+a^{4}\right) \tau\left|\left(1-\tau^{-a}\right) / a\right|^{2}$, and hence $J_{3} \ll$ $|G(1 / 2)| \ll\left(1+a^{4}\right) \tau\left|\left(1-\tau^{-a}\right) / a\right|^{2}+|G(3 / 8)|$. Using Lemma 4.4 and Proposition 5.2, we have

$$
\begin{aligned}
G(3 / 8) & \ll \tau^{-1 / 4-a}\left(\int_{\tau / 2}^{\tau}\left|\sum_{n \leq \tau} \frac{\sigma_{-a}(n)}{n^{3 / 8+i t}}\right|^{2} d t \int_{\tau / 2}^{\tau}\left|\sum_{n \leq \tau} \frac{\sigma_{a}(n)}{n^{3 / 8+i t}}\right|^{2} d t\right)^{1 / 2} \\
& \ll \tau^{-1 / 4-a}\left(\tau^{5 / 4} \Lambda^{*}(a, \tau) \cdot \tau^{5 / 4} \Lambda^{*}(-a, \tau)\right)^{1 / 2} \ll\left(1+|a|^{3}\right) \tau \Lambda(a, \tau),
\end{aligned}
$$

so that $J_{3} \ll\left(1+a^{4}\right)\left(\tau \Lambda(a, \tau)+\tau\left|\left(1-\tau^{-a}\right) / a\right|^{2}\right)$.

5.6. Evaluation of $J_{4}$ and $J_{5}$. Let

$$
G_{p q}(\sigma):=\int_{\tau / 2}^{\tau} S_{p}(a, s) F_{q}(a, 1-s) d t
$$

Let us evaluate $G_{13}(1 / 2)$. By Lemma 4.5 and (5.4), for $|\sigma-1 / 2| \leq 1 / 8$, we have $\mid F_{3}(a, 1-$ $s)\left|\ll \Gamma(|a|+2)\left(1+a^{2}\right) \tau^{\sigma}\right|\left(1-\tau^{-a}\right) / a \mid$ and $S_{1}(a, s) \ll\left(1+a^{2}\right) \tau^{1-\sigma}\left|\left(1-\tau^{-a}\right) / a\right|$. Hence $G_{13}(1 / 2) \ll\left|G_{13}(3 / 8)\right|+\left(1+|a|^{|a|}\right) \tau\left|\left(1-\tau^{-a}\right) / a\right|^{2}$, where

$$
G_{13}(3 / 8) \ll\left(\int_{\tau / 2}^{\tau}\left|S_{1}(a, 3 / 8+i t)\right|^{2} d t \int_{\tau / 2}^{\tau}\left|F_{3}(a, 5 / 8-i t)\right|^{2} d t\right)^{1 / 2} .
$$

Using

$$
\int_{(-3 / 4)}\left|\chi(w+5 / 8-i t) \chi(w+5 / 8+a-i t) \Gamma(w) \tau^{w} e^{\pi|v| / 4}\right|^{2}|d w| \ll \Gamma(2|a|+2) \tau^{1-2 a},
$$

we have

$$
\begin{gathered}
\Gamma(2|a|+2)^{-1} \int_{\tau / 2}^{\tau}\left|F_{3}(a, 5 / 8-i t)\right|^{2} d t \\
\ll \tau^{1-2 a} \int_{-\infty}^{\infty}\left(\int_{\tau / 2}^{\tau}\left|\sum_{n>\tau} \frac{\sigma_{a}(n) n^{i v}}{n^{9 / 8+i t}}\right|^{2} d t\right) e^{-\pi|v| / 2} d v \ll \tau^{3 / 4-2 a} \Lambda^{*}(-a, \tau) .
\end{gathered}
$$


From this estimate combined with

$$
\int_{\tau / 2}^{\tau}\left|S_{1}(a, 3 / 8+i t)\right|^{2} d t=\int_{\tau / 2}^{\tau}\left|\sum_{n \leq \tau} \frac{\sigma_{-a}(n)}{n^{3 / 8+i t}}\right|^{2} d t \ll \tau^{5 / 4} \Lambda^{*}(a, \tau),
$$

it follows that $G_{13}(3 / 8) \ll \Gamma(2|a|+2)^{1 / 2}(|a|+1) \tau \Lambda^{*}(a, \tau)$, which implies $G_{13}(1 / 2) \ll(1+$ $\left.|a|^{|a|}\right)\left(\tau \Lambda(a, \tau)+\tau\left|\left(1-\tau^{-a}\right) / a\right|^{2}\right)$. In treating $G_{22}(1 / 2)$ we use $\chi(s) \chi(s+a) \ll t^{1-2 \sigma-a}$, $\sum_{n \leq \tau} \sigma_{a}(n) / n^{1-s} \ll\left(1+a^{2}\right) \tau^{\sigma}\left|\left(\tau^{a}-1\right) / a\right|$ and

$$
\sum_{n>\tau} \frac{\sigma_{-a}(n) e^{-n / \tau}}{n^{1-s}} \ll\left(1+|a|^{|a|-4}\right) \sum_{n>\tau} \frac{\sigma_{-a}(n)}{n^{1-\sigma}}\left(\frac{\tau}{n}\right)^{|a|+1} \ll\left(1+|a|^{|a|-2}\right) \tau^{\sigma}\left|\frac{1-\tau^{-a}}{a}\right|
$$

for $|\sigma-1 / 2| \leq 1 / 8$. Then $G_{22}(1 / 2) \ll\left|G_{22}(5 / 8)\right|+\left(1+|a|^{|a|}\right) \tau\left|\left(1-\tau^{-a}\right) / a\right|^{2}$, where

$$
G_{22}(5 / 8) \ll \tau^{-\kappa-a}\left(\int_{\tau / 2}^{\tau}\left|\sum_{n \leq \tau} \frac{\sigma_{a}(n)}{n^{3 / 8-i t}}\right|^{2} d t \int_{\tau / 2}^{\tau}\left|\sum_{n>\tau} \frac{\sigma_{-a}(n) e^{-n / \tau}}{n^{3 / 8-i t}}\right|^{2} d t\right)^{1 / 2} .
$$

Using $\sum_{n>\tau} \sigma_{-a}(n)^{2} n^{-3 / 4} e^{-2 n / \tau} \ll\left(1+|a|^{2|a|-3}\right) \sum_{n>\tau} \sigma_{-a}(n)^{2} n^{-7 / 4-2|a|} \tau^{2|a|+1}$, we have $G_{22}(5 / 8) \ll\left(1+|a|^{2|a|}\right) \tau \Lambda(a, \tau)$, so that $G_{22}(1 / 2) \ll\left(1+|a|^{2|a|}\right)(\tau \Lambda(a, \tau)+$ $\left.\tau\left|\left(1-\tau^{-a}\right) / a\right|^{2}\right)$. The remaining integrals $G_{p q}(1 / 2)$ are similarly treated by considering $G_{11}(3 / 8), G_{12}(3 / 8), G_{14}(3 / 8), G_{21}(5 / 8), G_{23}(5 / 8)$ and $G_{24}(5 / 8)$. Thus we have $J_{4}=\overline{J_{5}} \ll\left(1+|a|^{2|a|}\right)\left(\tau \Lambda(a, \tau)+\tau\left|\left(1-\tau^{-a}\right) / a\right|^{2}\right)$. Combining (5.7) with the estimates for $J_{j}(2 \leq j \leq 6)$ given above, we immediately obtain Proposition 5.1.

\section{Proofs of the main results}

For $T \gg 1$, choose the positive integer $N_{0}:=\left[(3 / 4)(\log 2)^{-1} \log T\right]$. Then

$$
\bigcup_{0 \leq n \leq N_{0}}\left[2^{-n-1} T, 2^{-n} T\right]=\left[2^{-N_{0}-1} T, T\right], \quad T^{1 / 4} / 2 \leq 2^{-N_{0}-1} T \leq T^{1 / 4} .
$$

Split the integral $T \Psi(a, T)$ into two parts: $T \Psi(a, T)=V_{*}(a, T)+V_{0}(a, T)$ with

$$
\begin{aligned}
V_{*}(a, T) & :=\int_{1}^{2^{-N_{0}-1} T}|\zeta(1 / 2+i t) \zeta(1 / 2+a+i t)|^{2} d t \\
V_{0}(a, T) & :=\int_{2^{-N_{0}-1} T}^{T}|\zeta(1 / 2+i t) \zeta(1 / 2+a+i t)|^{2} d t
\end{aligned}
$$

The estimate $\zeta(1 / 2+i t) \ll t^{1 / 6}$ together with the convexity property gives $\zeta(\sigma+i t) \ll t^{1 / 4+\varepsilon}$ for $\sigma \geq 3 / 8, t \geq 1$. Hence, if $a \geq-1 / 8$,

$$
V_{*}(a, T) \ll\left(\int_{1}^{T^{1 / 4}}|\zeta(1 / 2+i t)|^{4} d t \int_{1}^{T^{1 / 4}}|\zeta(1 / 2+a+i t)|^{4} d t\right)^{1 / 2} \ll T .
$$


By the convexity property for an integral of $|\zeta(\sigma+i t)|^{4}$ (cf. $[6$, Lemma 8.3] or [16, §7.8]), if $a \leq-1 / 8$,

$$
V_{*}(a, T) \ll T^{(1-2 a) / 4+\varepsilon} \ll T^{-2 a+1 / 2} .
$$

Moreover by (6.1) and Proposition 5.1 with $T^{1 / 4} \leq \tau \leq T$, for $-T^{1 / 4} \leq a \leq 1 / 16$,

(6.4) $V_{0}(a, T)=\sum_{n=0}^{N_{0}} I\left(2^{-n-1} T, 2^{-n} T\right)=\frac{T}{2}\left(\sum_{n=0}^{N_{0}} 2^{-n} \Phi\left(a, 2^{-n} T\right)\right.$

$$
\begin{aligned}
& +(2 \pi)^{2 a} \lambda(a) \sum_{n=0}^{N_{0}} 2^{(2 a-1) n} T^{-2 a} \Phi\left(-a, 2^{-n} T\right)\left(1+O\left(\left(2^{-n} T\right)^{-1 / 4}\right)\right) \\
& \left.+\sum_{n=0}^{N_{0}} 2^{-n} R_{0}\left(a, 2^{-n} T\right)\right) \\
& =\frac{T}{2}\left(\Phi_{N_{0}}(a, T)+(2 \pi)^{2 a} \lambda(a) \widetilde{\Phi}_{N_{0}}(a, T)+R_{N_{0}}(a, T)\right)
\end{aligned}
$$

with

$$
\begin{aligned}
R_{N_{0}}(a, T) \ll & T^{-2 a-1 / 16} \sum_{n=0}^{N_{0}} 2^{(2 a-1) n}\left|\Phi\left(-a, 2^{-n} T\right)\right| \\
& +\left(|a|^{2|a|}+1\right)\left(|\Lambda|_{N_{0}}(a, T)+\left|\left(1-T^{-a}\right) / a\right|^{2}\right),
\end{aligned}
$$

since $\sum_{n=0}^{N_{0}} 2^{-n}\left|\left(1-\left(2^{-n} T\right)^{-a}\right) / a\right|^{2} \leq 2\left|\left(1-T^{-a}\right) / a\right|^{2}$. Here the implied constants are absolute; and $\Phi_{N}(a, x), \widetilde{\Phi}_{N}(a, x)$ and $|\Lambda|_{N}(a, x)$ are the quantities defined at the beginning of Section 4.

6.1. Proof of Theorem 2.1. By Lemma 4.6, $\Lambda(-a, \tau) \ll l_{3}(-a, \tau)$ for $T^{1 / 4} \leq \tau \leq$ $T$ if $|a|+(\log T)^{-1} \rightarrow 0$, so that, for $n \leq N_{0}$,

$$
\begin{aligned}
\Phi\left(-a, 2^{-n} T\right) & \ll \int_{1}^{2^{-n} T} \xi^{-1}|\Lambda(-a, \xi)| d \xi \ll \int_{1}^{2^{-n} T} \xi^{-1} l_{3}(-a, \xi) d \xi \\
& \ll a^{-4} \psi\left(-a, 2^{-n} T\right) \ll a^{-4} \psi(-a, T) .
\end{aligned}
$$

Furthermore $\left|\left(1-T^{-a}\right) / a\right|^{2} \cdot\left(a^{-4} \psi(a, T)\right)^{-1} \ll(\log T)^{-2}+a^{2}$. These estimates together with Lemmas 4.1 and 4.3 yield

$$
R_{N_{0}}(a, T) \ll a^{-4}\left(T^{-2 a} \psi(-a, T)+\psi(a, T)\right)\left(|a|+(\log T)^{-1}\right)
$$

with $T^{-2 a} \psi(-a, T)+\psi(a, T)=\phi(a \log T)$ as $|a|+(\log T)^{-1} \rightarrow 0$. Substitute this and $(2 \pi)^{2 a} \lambda(a)=1+O(a)$ into (6.4). Using Lemma 4.1 and (6.2), we obtain Theorem 2.1. 
6.2. Proof of Theorem 2.2. Suppose that $-T^{1 / 4} \leq a<0$ and that $|a \log T|^{-1}$ is sufficiently small. By Lemma 4.7 we have $\Lambda(a, \tau) \ll \Lambda_{0}(-a) \tau^{-2 a}$ and $\Lambda(-a, \tau) \ll \Lambda_{0}(-a)$ for $T^{1 / 4} \leq \tau \leq T$. Hence $\Phi\left(a, 2^{-n} T\right) \ll \Lambda_{0}(-a)|a|^{-1}\left(2^{-n} T\right)^{-2 a}$ and $\Phi\left(-a, 2^{-n} T\right) \ll$ $\Lambda_{0}(-a) \log T$ for $n \leq N_{0}$. Then, by Lemmas 4.2 and 4.3,

$$
\begin{aligned}
\Phi_{N_{0}}(a, T) & \ll \Lambda_{0}(-a)|a|^{-1} T^{-2 a} \ll \widetilde{\Phi}_{N_{0}}(a, T)|a \log T|^{-1}, \\
R_{N_{0}}(a, T) & \ll \Lambda_{0}(-a) T^{-2 a-1 / 16} \log T+\left(|a|^{2|a|}+1\right)\left(|\Lambda|_{N_{0}}(a, T)+a^{-2} T^{-2 a}\right) \\
& \ll\left(|a|^{2|a|}+1\right) \widetilde{\Phi}_{N_{0}}(a, T)(\log T)^{-1} .
\end{aligned}
$$

Using Lemma 4.2, (6.2), (6.3) and (6.4), we obtain the desired formula for $a<0$. For $0<$ $a \leq T^{1 / 4}$, we note the following:

$$
\begin{aligned}
T \Psi(a, T) & =\int_{1}^{T}|\zeta(1 / 2+i t) \zeta(1 / 2-a-i t) \chi(1 / 2+a+i t)|^{2} d t \\
& =(2 \pi)^{2 a} \int_{1}^{T}(t \Psi(-a, t))^{\prime} t^{-2 a}\left(1+O\left(t^{-1 / 2}\right)\right) d t \\
& =(2 \pi)^{2 a}(H(2 a, T)+O(H(2 a+1 / 2, T)))
\end{aligned}
$$

with

$$
H(\kappa, T):=T^{1-\kappa} \Psi(-a, T)+\kappa \int_{1}^{T} t^{-\kappa} \Psi(-a, t) d t
$$

since $(t \Psi(-a, t))^{\prime} \geq 0$. Substitution of the asymptotic expression for $\Psi(-a, T)$ with $a>0$ leads us to the second formula.

ACKNOWLEDGMENT. The author is grateful to the referee for helpful comments.

\section{References}

[ 1] S. BetTin, The second moment of the Riemann zeta-function with unbounded shifts, Int. J. Number Theory 6 (2010), 1933-1944.

[ 2 ] D. R. Heath-Brown, The fourth power moment of the Riemann zeta function, Proc. Lond. Math. Soc. (3) 38 (1979), 385-422.

[ 3 ] C. P. Hughes and M. P. Young, The twisted fourth moment of the Riemann zeta function, J. Reine Angew. Math. 641 (2010), 203-236.

[ 4 ] A. E. Ingham, Mean-value theorems in the theory of the Riemann zeta-function, Proc. Lond. Math. Soc. (2) 27 (1928), 273-300.

[ 5 ] M. Ishibashi and S. KAnemitsu, Some asymptotic formulas of Ramanujan, Analytic number theory (Tokyo, 1988), Lecture Notes in Math., 1434, Springer, Berlin, 1990, 149-167.

[ 6] A. IVIĆ, The Riemann Zeta-function, Wiley, New York, 1985.

[ 7 ] I. KIUCHI, On an exponential sum involving the arithmetic function $\sigma_{a}(n)$, Math. J. Okayama Univ. 29 (1987), 193-205.

[ 8 ] H. KÖSTERS, On the occurrence of the sine kernel in connection with the shifted moments of the Riemann zeta function, J. Number Theory 130 (2010), 2596-2609. 
[ 9 ] H. L. Montgomery and R. C. Vaughan, Hilbert's inequality, J. Lond. Math. Soc. 8 (1974), 73-82.

[10] Y. Мотонаsнi, Spectral Theory of the Riemann Zeta-function, Cambridge Tracts Math. 127, Cambridge University Press, Cambridge, 1997.

[11] Y.-F. S. PÉtermann and J. WU, On some asymptotic formulæ of Ramanujan, Math. Nachr. 242 (2002), 165-178.

[12] K. RAmAChAndRA, A simple proof of the mean fourth power estimate for $\zeta\left(\frac{1}{2}+i t\right)$ and $L\left(\frac{1}{2}+i t, \chi\right)$, Ann. Scuola Norm. Sup. Pisa 1 (1974), 81-97.

[13] K. RamachandRA, Application of a theorem of Montgomery and Vaughan to the zeta-function, J. Lond. Math. Soc. 10 (1975), 482-486.

[14] K. RAmachandra and A. SankARAnARAYAnAn, On an asymptotic formula of Srinivasa Ramanujan, Acta Arith. 109 (2003), 349-357.

[15] S. Shimomura, Shifted fourth moment of the Riemann zeta-function, Acta Math. Hungar. 137 (2012), 104129.

[16] E. C. Titchmarsh, The Theory of the Riemann Zeta-function, Second edition, The Clarendon Press, Oxford University Press, New York, 1986.

Present Address:

DEPARTMENT OF MATHEMATICS,

KEIO UNIVERSITY,

3-14-1, HiYOSHI, KOHOKU-KU, YOKOHAMA, 223-8522 JAPAN.

e-mail: shimomur@math.keio.ac.jp 